\title{
Spin Self-Rephasing and Very Long Coherence Times in a Trapped Atomic Ensemble
}

\author{
C. Deutsch, ${ }^{1}$ F. Ramirez-Martinez,${ }^{2}$ C. Lacroûte ${ }^{2}$ F. Reinhard, ${ }^{1, *}$ T. Schneider, ${ }^{1 \dagger}$ J. N. Fuchs, ${ }^{3}$ F. Piéchon, ${ }^{3}$ F. Laloë, \\ J. Reichel, ${ }^{1}$ and P. Rosenbusch ${ }^{2, \$}$ \\ ${ }^{1}$ Laboratoire Kastler Brossel, ENS, UPMC, CNRS, 24 rue Lhomond, 75005 Paris, France \\ ${ }^{2}$ LNE-SYRTE, Observatoire de Paris, UPMC, CNRS, 61 av de l'Observatoire, 75014 Paris, France \\ ${ }^{3}$ Laboratoire de Physique des Solides, CNRS UMR 8502, Univ. Paris-Sud, 91405 Orsay, France \\ (Received 24 March 2010; revised manuscript received 28 May 2010; published 6 July 2010)
}

\begin{abstract}
We perform Ramsey spectroscopy on the ground state of ultracold ${ }^{87} \mathrm{Rb}$ atoms magnetically trapped on a chip in the Knudsen regime. Field inhomogeneities over the sample should limit the 1/e contrast decay time to about $3 \mathrm{~s}$, while decay times of $58 \pm 12 \mathrm{~s}$ are actually observed. We explain this surprising result by a spin self-rephasing mechanism induced by the identical spin rotation effect originating from particle indistinguishability. We propose a theory of this synchronization mechanism and obtain good agreement with the experimental observations. The effect is general and may appear in other physical systems.
\end{abstract}

PACS numbers: 05.30. $-\mathrm{d}$, 06.30.Ft, 32.30. $-\mathrm{r}, 67.85 .-\mathrm{d}$

In atomic clocks and other precision techniques based on atomic spin manipulation [1], a central requirement is to preserve the coherence of a state superposition over long times. Understanding how coherence decays in a given system is important for these applications, and is a touchstone of understanding its dynamics. In trapped ensembles, an inhomogeneous shift $\Delta(\mathbf{r})$ of the transition frequency occurs due to the trapping potential and to atomic interactions. Different atoms explore different regions of this shift landscape, and so their spins precess at different rates. This leads to dephasing at a rate determined by the characteristic inhomogeneity $\Delta_{0}$ of $\Delta(\mathbf{r})$ over the ensemble. Various mechanisms have been exploited to reduce this dephasing. Examples are "magic fields" that strongly reduce the field dependence for a specific transition $[2,3]$, or the mutual compensation scheme successfully employed in ultracold ${ }^{87} \mathrm{Rb}$ [4], where the trap-induced inhomogeneity can be adjusted to nearly cancel the collisional mean-field inhomogeneity. All such mechanisms, however, including the motional narrowing well known in nuclear magnetic resonance, have in common that the dephasing is merely slowed down, but never reversed, and the transverse polarization remains a steadily decreasing function of time.

Here we present measurements on a trapped ensemble of ${ }^{87} \mathrm{Rb}$ atoms with two internal levels equivalent to a spin $1 / 2$. Atomic interactions cause a spontaneous rephasing of the spins, observed as a much longer decay time and revivals of Ramsey contrast. We are also able to extend the coherence time by more than an order of magnitude beyond the 2 to $3 \mathrm{~s}$ previously achieved on this system $[3,5]$. We explain these remarkable results by a very general mechanism based on the identical spin rotation effect (ISRE) that occurs during collisions in the forward direction between two identical particles [6] - an equivalent description can be given in terms of the exchange meanfield experienced by the atoms [7]. This effect is known to cause transient spin waves $[4,8-14]$, a deleterious phe- nomenon if one is interested in long coherence times. In contrast to those experiments, however, we are working in a regime where both (i) the ISRE rate (exchange rate) $\omega_{\text {ex }} / 2 \pi=2 \hbar\left|a_{01}\right| \bar{n} / m$ is larger than the inhomogeneity $\Delta_{0}$ and (ii) the rate of lateral elastic collisions $\gamma_{c}=$ $(32 \sqrt{\pi} / 3) a_{01}^{2} \bar{n} v_{T}$ is much lower than both the trap frequencies (Knudsen regime) and $\omega_{\mathrm{ex}}$. Here, $a_{01}$ is the relevant scattering length [15], $\bar{n}$ the average density and $v_{T} \equiv \sqrt{k_{B} T / m}$ the thermal velocity for atomic mass $m$. In this regime, we find that the ISRE introduces efficient synchronization between atoms with different spin precession rates. Indeed, solving a kinetic equation for the spin variables based on the ISRE, we obtain good agreement with the data. These findings are reminiscent of earlier calculations for a trapped gas which predict localized polarization revivals [12] and synchronization within spatial domains [10] in a different (hydrodynamic) regime.

We start with a simple model (Fig. 1), where as in [13] we divide the atoms into two classes having fast and slow transverse spin precession rates. Whether an atom is in the fast or slow class depends on the average of $\Delta(\mathbf{r})$ it experiences. In the second experiment below, for example, this depends on its orbital energy. If the class-changing events
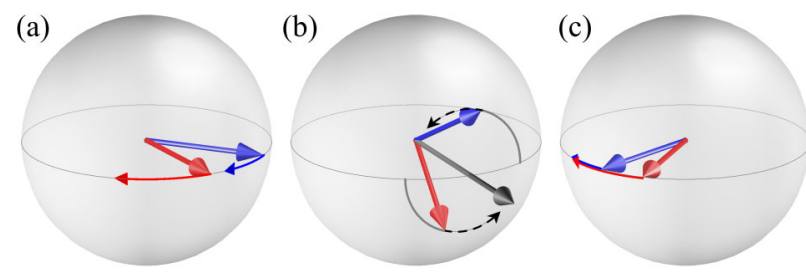

FIG. 1 (color online). Two classes of atoms (red and blue) precess at different rates. Their Bloch vectors were initially parallel, but have started to dephase (a). The ISRE then makes both vectors rotate around their sum (grey) (b). When this rotation reaches $\pi$, the fast-precessing spin (red) lags behind the slow one (blue), which tends to rephase them as in a spin echo (c). 
are rare $\left(\gamma_{c}<\omega_{\mathrm{ex}}\right)$, an atom remains in its class for a long time and the two classes start to dephase. The effect of the ISRE is then simply to make the spin polarizations of the slow and fast class turn around their sum, reversing their phase differences on the time scale of $\pi / \omega_{\mathrm{ex}}$. When this rotation reaches $\pi$, the two transverse polarizations are exchanged, while each of them continues to precess at the same rate as before, so that they start to rephase.

The ISRE does not change the sum of the polarizations: it just reverts the correlation between the spin directions and their precession rate. This initiates the rephasing process that increases the transverse polarization. Of course, in reality dephasing and refocusing occur simultaneously; the result is a synchronization mechanism that is analogous to a negative feedback effect. It is also reminiscent of a spin echo, but here the rephasing of the spins is due to an internal effect instead of externally applied rf pulses.

For a self-rephasing regime to occur, the ISRE must revert the spins before lateral collisions cause atoms to change class-that is, $\omega_{\mathrm{ex}} / \pi>\gamma_{c}$-and before the dephasing reaches $\pi, \omega_{\mathrm{ex}}>\Delta_{0}$ (otherwise it accelerates the dephasing). If $\omega_{\mathrm{ex}} \gg \Delta_{0}$, any dephasing will be immediately refocused, leading to tight synchronization and long coherence times; if $\omega_{\mathrm{ex}} \gtrsim \Delta_{0}$, a significant phase spread can occur before the spins rephase, leading to loss and revival of the total polarization. Performing two experiments, we have indeed observed these behaviors, and found good agreement with our quantitative calculations.

We perform Ramsey spectroscopy on the $|0\rangle \equiv$ $\left|F=1, m_{F}=-1\right\rangle$ to $|1\rangle \equiv\left|F=2, m_{F}=1\right\rangle$ hyperfine transition of ${ }^{87} \mathrm{Rb}$ atoms magnetically trapped on a chip. The differential Zeeman shift $\Delta_{Z}(\mathbf{r})$ is quadratic in magnetic field around a "magic" value $B_{m}=3.228917$ (3) G [3] and quartic in position. The $s$-wave scattering lengths $a_{00}, a_{11}$ and $a_{01}$ differ by less than 5\% [16], so that the mean-field shift of the transition frequency is already small with $\Delta_{\mathrm{mf}}(\mathbf{r}) / 2 \pi=-0.4 \mathrm{~Hz} \times n(\mathbf{r}) / 10^{12} \mathrm{~cm}^{-3}$ for an equal superposition of $|0\rangle$ and $|1\rangle$, where $n(\mathbf{r})$ is the local density. Additionally, we employ the mutual compensation technique [3]: adjusting the magnetic field at the trap center $B_{0}$ to a value slightly below $B_{m}$ leads to a standard deviation of $\Delta(\mathbf{r}) \equiv \Delta_{Z}(\mathbf{r})+\Delta_{\mathrm{mf}}(\mathbf{r})$ averaged over the atomic cloud that is of order $\Delta_{0}=2 \pi \times 0.08 \mathrm{~Hz}$ [17].

The experiment is designed with the goal of a "trappedatom clock on a chip" (TACC), aiming at a stability in the lower $10^{-13} \mathrm{~s}^{-1 / 2}$ range. The setup is described in detail in [18], and is similar to that of [19]. It incorporates a twolayer atom chip with a coplanar waveguide (CPW). Atom preparation involves the usual laser and evaporative cooling steps [20]. Within less than $10 \mathrm{~s}$ we obtain $N_{\text {at }}=5 \times$ $10^{3}$ to $10^{5}$ trapped atoms in state $|0\rangle$ at a temperature of $T=175(6) \mathrm{nK}$, which is at least $30 \mathrm{nK}$ above the onset of Bose Einstein condensation (BEC) for our densities. We use a two-photon, microwave (MW) and radio frequency (rf) excitation to drive the clock transition. The MW frequency is detuned $\sim 500 \mathrm{kHz}$ above the $|0\rangle$ to
$\left|F=2, m_{F}=0\right\rangle$ transition. The detuning from the twophoton resonance is set to $\Delta_{R} / 2 \pi=3.6 \mathrm{~Hz}$. The MW is generated by a home-built synthesizer having very low phase noise [21]; a commercial direct digital synthesizer provides the rf. Both are locked to a hydrogen maser of relative frequency stability $10^{-13} \tau^{-1 / 2}$ up to $1000 \mathrm{~s}$ [22]. Both signals are injected into structures on the chip with powers $\sim 0 \mathrm{dBm}(\mathrm{MW}) \sim 5 \mathrm{dBm}(\mathrm{rf})$. Trapped below the CPW at a distance $z_{0}$, the atoms interact with its evanescent field and perform two-photon Rabi oscillations at a rate $\Omega_{R}$. In each cycle, we detect the number of atoms in both clock states, $N_{1}$ and $N_{0}$, by absorption imaging without and with repump light after a time of flight of 9 and $13 \mathrm{~ms}$, respectively. We use absorption imaging close to saturation intensity [23]. Intensity and magnification are carefully calibrated, and we have verified that cross talk between the detected $N_{0}$ and $N_{1}$ is negligible. We estimate the calibration error on the absolute number of atoms to $5 \%$. The statistical error is 140 atoms per state.

In a first experiment, the magnetic trap has frequencies $\left\{\omega_{x}, \omega_{y}, \omega_{z}\right\} / 2 \pi=\{32(1), 97.5(2.5), 121(1)\} \mathrm{Hz}$; for this trap $z_{0}=156 \mu \mathrm{m}$ and $\Omega_{R}=2 \pi \times 164 \mathrm{~Hz}$. $B_{0}$ is optimized roughly by maximizing the fringe contrast after a Ramsey time $T_{R}=2 \mathrm{~s}$. This leads to $B_{0}=3.1626(7) \mathrm{G}$, as measured by rf-induced atom loss on a small BEC $\left(N_{\mathrm{at}} \sim\right.$ 3000 ). The two $\pi / 2$ pulses, spaced by a variable Ramsey time $T_{R}$, are applied at the end of a period of fixed length of $T_{T}=5.02 \mathrm{~s}$, and preceded by a holding time $T_{H}=T_{T}-$ $T_{R}$ during which the atoms are in $|0\rangle$. The transition probability $N_{1} /\left(N_{0}+N_{1}\right)$ is plotted in Fig. 2(a) as a function of $T_{R}$. The contrast at $T_{R}=0$ is $89 \%$, probably limited by a small inhomogeneity of the Rabi frequency. At $T_{R}=$ $5 \mathrm{~s}$ the contrast still remains above $82 \%$. Assuming exponential decay, the $1 / e$ time is $58 \pm 12 \mathrm{~s}$, much longer than the $2.75 \mathrm{~s}$ predicted from the mutual compensation scheme [17]. The true dephasing is even slower, since the atom loss rates $\gamma_{0}$ and $\gamma_{1}$ of $|0\rangle$ and $|1\rangle$ differ [Fig. 2(b)]; asymmetric loss leads to a decay of Ramsey contrast that is independent of dephasing.

The very long coherence time can be understood as a tight synchronization due to self-rephasing. The density $\bar{n} \approx 1$ (from now on in units of $10^{12} \mathrm{~cm}^{-3}$ ) gives an ISRE rate $\omega_{\mathrm{ex}} / 2 \pi \approx 8 \mathrm{~Hz}$, much larger than the inhomogeneity $\Delta_{0} / 2 \pi \approx 0.08 \mathrm{~Hz}$, so that the fast and slow spins are swapped when the dephasing is still small. Moreover, since $\gamma_{c} \approx 2 \mathrm{~s}^{-1}$, the correlations between precession rate and accumulated dephasing remain intact over long times.

In a second experiment, we deliberately increase $\Delta_{0}$ by detuning $B_{0}$ away from the optimal value. We choose $B_{0}=$ 3.7562(9) G, such that the inhomogeneity of the transverse precession rate is now well approximated by a parabolic spatial dependence $\Delta(\mathbf{r}) \approx \Delta_{0}\left(\left(x / x_{T}\right)^{2}+\left(y / y_{T}\right)^{2}+\right.$ $\left.\left(z / z_{T}\right)^{2}\right)$ with $\left\{x_{T}, y_{T}, z_{T}\right\} \equiv v_{T} /\left\{\omega_{x}, \omega_{y}, \omega_{z}\right\}$. Here, $\Delta_{0}=$ $\left(x_{T}^{2} / 2\right)\left\langle\partial_{x}^{2} \Delta\right\rangle \approx 2 \pi(1.2+0.1 \times \bar{n}) \mathrm{Hz}$ by averaging over the gaussian density profile $n(\mathbf{r})$. Note that $\Delta_{0}$ is also linear in temperature, which is constant in our experiment. 

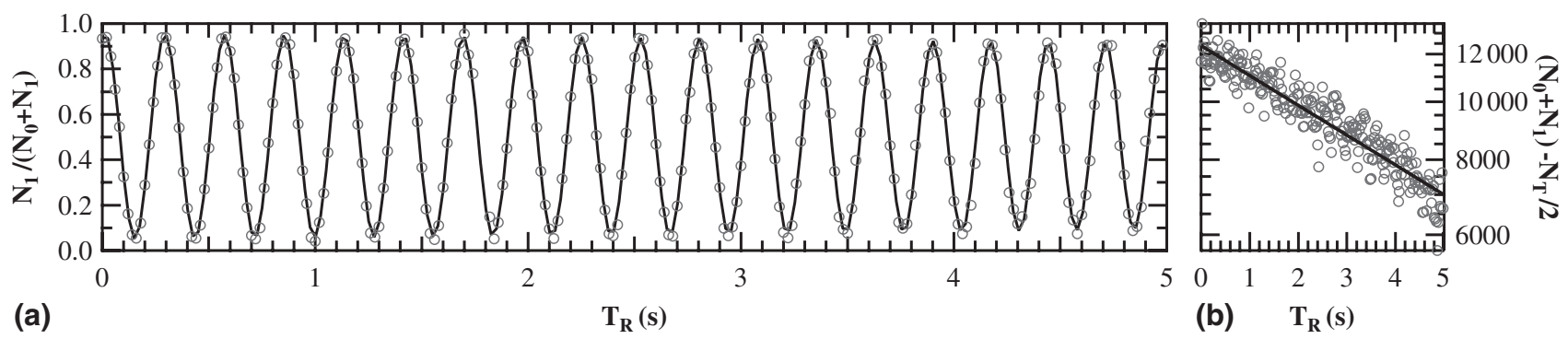

FIG. 2. First experiment: (a) Ramsey fringes in the time domain for $\Delta_{0} / 2 \pi \approx 0.08 \mathrm{~Hz}$. The normalized transition probability is plotted as a function of the Ramsey free evolution time. The initial contrast is $89 \%$, probably limited by an inhomogeneous Rabi frequency. At $T_{R}=5 \mathrm{~s}$, the contrast is $82 \%$ resulting in a $1 / e$ time of $58 \pm 12 \mathrm{~s}$. (b) Total number of detected atoms. Although the total time $T_{T}$ is kept constant, this number depends on $T_{R}$ because the loss rates of $|0\rangle$ and $|1\rangle$ differ. The data is fitted by $N_{0}+N_{1}=$ $N_{T} / 2\left(1+e^{-T_{R} / \tau}\right)$ with $N_{T}=24.8(2) \times 10^{3}$ atoms and $\tau=8.7(2) \mathrm{s}$.

The new $B_{0}$ leads to trap frequencies $\left\{\omega_{x}, \omega_{y}, \omega_{z}\right\} / 2 \pi=$ $\{31.30(5), 92.0(5), 117.0(3)\} \mathrm{Hz} ; z_{0}=151 \mu \mathrm{m}$ and $\Omega_{R}=$ $2 \pi \times 83 \mathrm{~Hz}$. The total time is reduced to $T_{T}=1 \mathrm{~s}$ and the Ramsey time is varied between 15 and $500 \mathrm{~ms}$. For each $T_{R}$, we vary the detuning $\Delta_{R}$ in 30 steps and extract the fringe contrast. We repeat the measurement for different $\bar{n}$ spanning 1 order of magnitude. To vary $\bar{n}$, we vary the atom number by changing the MOT loading time. (We have checked that the temperature is independent of $\bar{n}$ within our measurement precision.) The lowest density is $\bar{n}=$ 0.2 , which corresponds to $\omega_{\mathrm{ex}} / 2 \pi=1.5 \mathrm{~Hz}$ and $\gamma_{c}=$ $0.4 \mathrm{~s}^{-1}$.

The results are plotted in figure. 3. All datasets show initial rapid contrast decay between 0 and $50 \mathrm{~ms}$. For $\bar{n}=$ 0.2 , it vanishes completely at $\sim 200 \mathrm{~ms}$. Increasing the density stabilizes the contrast beyond $200 \mathrm{~ms}$ and leads to revivals. The contrast improves with increasing density, indicating an interaction effect. The time of the first revival depends on density with $T_{\text {revival }} \approx-0.02 \mathrm{~s}+0.3 \mathrm{~s} / \bar{n}$. This agrees to within a factor of 2 with the estimation $2 \pi / \omega_{\mathrm{ex}}=0.13 \mathrm{~s} / \bar{n}$ from our simple model above.

To interpret this data, we now perform a quantitative calculation. As in [11,12], the motion in the trap is treated semiclassically, while a full quantum treatment of the spin variables is included in a kinetic equation for a density operator $\hat{\rho}(\mathbf{r}, \mathbf{p}, t)$, characterized by the usual Bloch vector $\mathbf{S}=\operatorname{Tr}(\hat{\rho} \hat{\boldsymbol{\sigma}} / 2)$. The vector components of $\mathbf{S}$ and the Pauli matrices $\hat{\boldsymbol{\sigma}}$ point along the three unit vectors of the Bloch sphere $\left\{\mathbf{u}_{\perp 1}, \mathbf{u}_{\perp 2}, \mathbf{u}_{\|}\right\}$. To describe the harmonic oscillator in phase space, instead of the usual $\left\{x, p_{x}\right\}$ (and similarly for $y$ and $z)$, we use energy-angle variables $\left\{E_{x}=\left(x^{2}+\right.\right.$ $\left.\left.p_{x}^{2}\right) / 2, \alpha_{x}=\arctan \left(p_{x} / x\right)\right\}$, where $E_{x}, x$ and $p_{x}$ are, respectively, in units of $k_{B} T, x_{T}$ and $m v_{T}$. As the oscillatory motion is fast compared to the spin dynamics $\left(\omega_{x, y, z} \gg\right.$ $\left.\omega_{\text {ex }}, \Delta_{0}, \gamma_{c}\right)$, we average $\mathbf{S}(\mathbf{E}, \boldsymbol{\alpha}, t)$ over the angles $\boldsymbol{\alpha}=$ $\left(\alpha_{x}, \alpha_{y}, \alpha_{z}\right)$ and obtain an equation for a spin density $\mathbf{S}(\mathbf{E}, t)$ in energy space only, where $\mathbf{E}=\left(E_{x}, E_{y}, E_{z}\right)$ [24]. This description is inspired by that developed in [9] for a quasi-1D gas in the Knudsen regime, but generalized to $3 \mathrm{D}$ and with the effect of lateral collisions giving rise to a damping term $\propto \gamma_{c}$. At this stage, the equation still depends on each $E_{i}$ separately through the frequency shift $\Delta(\mathbf{E})$. However, since our $\Delta(\mathbf{r})$ is parabolic in $\mathbf{r}$, it becomes $\Delta(E)=\Delta_{0} E$ when averaged over the angles and only depends on the total energy $E=E_{x}+E_{y}+E_{z}$. This allows us to write an equation for an energy-isotropic spin density $\mathbf{S}(E, t)$ :

$$
\begin{aligned}
& \partial_{t} \mathbf{S}(E, t)+\gamma_{c}[\mathbf{S}(E, t)-\overline{\mathbf{S}}(t)] \\
& \approx\left[\Delta(E) \mathbf{u}_{\|}+\omega_{\mathrm{ex}} \int_{0}^{\infty} d E^{\prime} \frac{E^{\prime 2}}{2} e^{-E^{\prime}} K\left(E, E^{\prime}\right) \mathbf{S}\left(E^{\prime}, t\right)\right] \\
& \quad \times \mathbf{S}(E, t),
\end{aligned}
$$

where $\overline{\mathbf{S}} \equiv \int_{0}^{\infty} d E \frac{E^{2}}{2} e^{-E} \mathbf{S}(E)$ is the average spin and $E^{2} / 2$ is the $3 \mathrm{D}$ harmonic oscillator density of states. Upon angle-

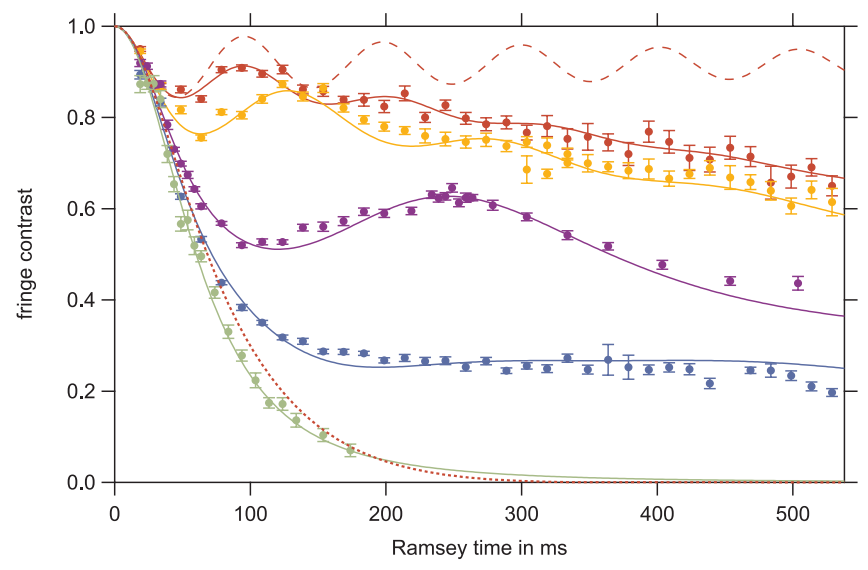

FIG. 3 (color online). Second experiment: Ramsey fringe contrast as a function of $T_{R}$ for increased inhomogeneity $\Delta_{0} / 2 \pi \approx 1.2 \mathrm{~Hz}$. From bottom to top, colors correspond to densities $\quad \bar{n}=\{0.2,0.8,1.1,1.9,2.6\} \times 10^{12} \mathrm{~cm}^{-3} \quad\left(\right.$ or $\quad N_{\text {at }}=$ $\left.\{7.9,28,41,70,95\} \times 10^{3}\right)$. For each $\bar{n}$, the data is normalized to the initial contrast at $T_{R}=0$. Solid lines are numerical solutions of the kinetic equation for $\Delta_{0} / 2 \pi \approx 2 \mathrm{~Hz}$ taking into account the ISRE $\left(\omega_{\text {ex }} / 2 \pi \approx 4.5 \mathrm{~Hz} \times \bar{n}\right)$ and lateral collisions $\left(\gamma_{c} \approx 2.1 \mathrm{~s}^{-1} \times \bar{n}\right)$. The red dotted line is a calculation for $\bar{n}=2.6$ (same density as the red data points) with lateral collisions $\left(\gamma_{c}=5 \mathrm{~s}^{-1}\right)$, but without ISRE. The red dashed line is a calculation without lateral collisions but with ISRE $\left(\omega_{\mathrm{ex}} / 2 \pi=12 \mathrm{~Hz}\right)$. 
averaging, the spin mean-field, which is local in position, becomes long-ranged in energy space with a kernel $K\left(E, E^{\prime}\right)$. In 1D, this kernel $K_{1}\left(E, E^{\prime}\right) \approx\left[\max \left(E, E^{\prime}\right) \mid E-\right.$ $\left.E^{\prime} \mid\right]^{-1 / 4}$ [9]. In 3D, it is also long-ranged but cumbersome to use. For simplicity, when solving (1) numerically, we approximate [25] it as infinite-ranged $K\left(E, E^{\prime}\right) \approx 1$. Equation (1) does not include atom loss. It contains three experimentally tunable parameters: the inhomogeneity $\Delta_{0}(T, \bar{n}, \ldots)$, the ISRE rate $\omega_{\mathrm{ex}} \propto\left|a_{01}\right| \bar{n}$ and the lateral collision rate $\gamma_{c} \propto a_{01}^{2} \bar{n} \sqrt{T}$.

To compare this theory to the experiment, we first consider the lowest-density data set $(\bar{n}=0.2)$, where both the ISRE and lateral collisions are negligible $\left(\Delta_{0} \gg \omega_{\mathrm{ex}}, \gamma_{c}\right)$. When they vanish, the kinetic equation is easily solved analytically. For an initial condition $\mathbf{S}(E, 0)=\mathbf{u}_{\perp 1}$, the contrast is $|\overline{\mathbf{S}}(t)|=\left(1+\left(\Delta_{0} t\right)^{2}\right)^{-3 / 2}$. Fitting the experimental contrast with this result, we find, $\Delta_{0} / 2 \pi \approx 2 \mathrm{~Hz}$, not far from its expected value $\Delta_{0} / 2 \pi \approx 1.2 \mathrm{~Hz}$. We then numerically solve Eq. (1) for the other densities $\bar{n}=$ $\{0.8,1.1,1.9,2.6\}$. Choosing $\Delta_{0} / 2 \pi$ within $5 \%$ of the above $2 \mathrm{~Hz}, \gamma_{c}=(32 \sqrt{\pi} / 3) a_{01}^{2} \bar{n} v_{T} \approx 2.1 \mathrm{~s}^{-1} \times \bar{n}$ as predicted and $\omega_{\mathrm{ex}} / 2 \pi \approx 0.6 \times 2 \hbar\left|a_{01}\right| \bar{n} / m \approx 4.5 \mathrm{~Hz} \times \bar{n}$ reproduces all data well (solid lines in Fig. 3). The renormalization of the ISRE rate by a factor 0.6 results from the overestimation of the synchronization effect through the infinite-range approximation.

If we set $\omega_{\text {ex }}=0$, the theory predicts short coherence time and no revivals (dotted line in Fig. 3) for all densities, confirming that the ISRE is responsible for the revivals. We also solve the $\bar{n}=2.6$ case without lateral collisions, $\gamma_{c}=$ 0: the dashed line in Fig. 3 shows an initial drop and revival, but continues to oscillate around a constant value. The lateral collisions are therefore responsible for the slow decay of the contrast at long times. We note that, in contrast to the mechanism of [10], here the spin synchronization does not result from a simple compensation of the inhomogeneous longitudinal field $\Delta(E) \mathbf{u}_{\|}$by the exchange mean field $\omega_{\mathrm{ex}} \overline{\mathbf{S}}(t)$ as they are orthogonal. Also, while Ref. [26] discusses the effects of the inhomogeneities of the probe field during the $\pi / 2$ pulses, our work deals with the effects of the static field between them (free precession).

In conclusion, the observed spin self-rephasing and synchronization are relatively robust when the inhomogeneity to compensate is not too large: in the first experiment, a large orientation is still present after more than 10 velocity-changing collisions. The effect may occur in any sample where the exchange rate $\omega_{\text {ex }}$ is larger than the characteristic dephasing rate $\Delta_{0}$ and the lateral collision rate $\gamma_{c}$. The ratio between these rates is tunable through the temperature dependence of $\omega_{\mathrm{ex}} / \gamma_{c}$ and through Feshbach resonances. It should be interesting to investigate whether the mechanism occurs in systems such as optical lattice clocks [2].

This work was supported from the EURYI grant "Integrated Quantum Devices", the Institut Francilien pour la Recherche sur les Atomes Froids (IFRAF), and the Delegation Generale de l'Armement (DGA), contract 07.34.005.

*Present address: 3. Physikal. Institut, Universität Stuttgart, 70770 Stuttgart, Germany.

${ }^{\dagger}$ Present address: Heinrich-Heine Universität Düsseldorf, 40225 Düsseldorf, Germany.

Peter.Rosenbusch@obspm.fr

[1] S. Chu, Nature (London) 416, 206 (2002).

[2] H. Katori, M. Takamoto, V.G. Pal'chikov, and V.D. Ovsiannikov, Phys. Rev. Lett. 91, 173005 (2003).

[3] D. M. Harber, H. J. Lewandowski, J. M. McGuirk, and E. A. Cornell, Phys. Rev. A 66, 053616 (2002).

[4] H. J. Lewandowski, D. M. Harber, D. L. Whitaker, and E. A. Cornell, Phys. Rev. Lett. 88, 070403 (2002).

[5] P. Treutlein, P. Hommelhoff, T. Steinmetz, T. W. Hänsch, and J. Reichel, Phys. Rev. Lett. 92, 203005 (2004).

[6] C. Lhuillier and F. Laloë, J. Phys. (Paris) 43, 197 (1982).

[7] E. P. Bashkin, JETP Lett. 33, 8 (1981).

[8] X. Du, L. Luo, B. Clancy, and J.E. Thomas, Phys. Rev. Lett. 101, 150401 (2008).

[9] X. Du, Y. Zhang, J. Petricka, and J. E. Thomas, Phys. Rev. Lett. 103, 010401 (2009).

[10] M. Ö. Oktel and L. S. Levitov, Phys. Rev. Lett. 88, 230403 (2002).

[11] J. N. Fuchs, D. M. Gangardt, and F. Laloë, Phys. Rev. Lett. 88, 230404 (2002).

[12] J. E. Williams, T. Nikuni, and C. W. Clark, Phys. Rev. Lett. 88, 230405 (2002).

[13] F. Piéchon, J. N. Fuchs, and F. Laloë, Phys. Rev. Lett. 102, 215301 (2009).

[14] S. S. Natu and E. J. Mueller, Phys. Rev. A 79, 051601(R) (2009).

[15] This value of $\gamma_{c}$ assumes $a_{00} \approx a_{11} \approx a_{01}$, as in our experiment.

[16] E. G. M. van Kempen, S. J. J. M. F. Kokkelmans, D. J. Heinzen, and B. J. Verhaar, Phys. Rev. Lett. 88, 093201 (2002).

[17] P. Rosenbusch, Appl. Phys. B 95, 227 (2009).

[18] C. Lacroute, F. Reinhard, F. Ramirez-Martinez, C. Deutsch, T. Schneider, J. Reichel, and P. Rosenbusch, IEEE Trans. Ultrason. Ferroelectr. Freq. Control 57, 106 (2010).

[19] P. Böhi, M. F. Riedel, J. Hoffrogge, J. Reichel, and T. W. Hänsch, Nature Phys. 5, 592 (2009).

[20] W. Hänsel, P. Hommelhoff, T. W. Hänsch, and J. Reichel, Nature (London) 413, 498 (2001).

[21] F. Ramirez-Martinez, M. Lours, P. Rosenbusch, F. Reinhard, and J. Reichel, IEEE Trans. Ultrason. Ferroelectr. Freq. Control 57, 88 (2010).

[22] D. Chambon, S. Bize, M. Lours, F. Narbonneau, H. Marion, A. Clairon, G. Santarelli, A. Luiten, and M. Tobar, Rev. Sci. Instrum. 76, 094704 (2005).

[23] G. Reinaudi, T. Lahaye, Z. Wang, and D. Guéry-Odelin, Opt. Lett. 32, 3143 (2007).

[24] Details of the derivation will be given elsewhere.

[25] We checked that in 1D this approximation is very good.

[26] K. Gibble, Phys. Rev. Lett. 103, 113202 (2009). 